\title{
Aqueous Two Phase Extraction for the Recovery of 1,3-Propanediol from Its Aqueous Solutions
}

\author{
Min Hee Chung ${ }^{1}$, Yeon Ki Hong ${ }^{1}$, Hyoung Wook Lee ${ }^{2}$, Sung-Jun Park ${ }^{3}$ \\ ${ }^{1}$ Department of Chemical and Biological Engineering, Korea National University of Transportation, Chungju, Chungbuk, Korea \\ ${ }^{2}$ Department of Energy System Engineering, Korea National University of Transportation, Chungju, Chungbuk Korea \\ ${ }^{3}$ Department of Mechanical Engineering, Korea National University of Transportation, Chungju, Chungbuk Korea \\ Email: hongyk@ut.ac.kr, hwlee@ut.ac.kr, park@ut.ac.kr
}

Received 2012

\begin{abstract}
As the biodiesel production is rapidly enhanced, the crude glycerol, which is by-product of biodiesel processes, is state of surplus. 1,3-PDO (1,3-propanediol), a valuable monomer of poly(trimethylene terephthalate) (PTT), can be produced from the fermentation process using crude glycerin as a carbon source. For the economic biological production of 1,3-PDO, the low cost and high efficient separation processes is essential. In this study, aqueous two-phase system composed of various hydrophilic alcohols and salt was used as a primary separation step for 1,3-PDO. It was found that the aqueous two-phase systems are easily formed with decreasing of the polarity of alcohols. The extraction efficiency is proportional to the polarity of alcohols. In case of methanol or ethanol/K2HPO4, the extraction efficiency was more than $90 \%$. It was concluded that the aqueous two-phase extraction using methanol or ethanol/K2HPO4 can be applied for the primary separation of 1,3-PDO as an alternative to a conventional primary separation processes.
\end{abstract}

Keywords: 1,3-Propanediol; Alcohols; Phase Separation; Extraction Efficiency

\section{Introduction}

Over the last decade, biodiesel has emerged as an alternative fuel of fossil diesel. However, the production of biodiesel is not profitable without government subsidies due to its high production cost. For the economic production of biodiesel, finding new applications of glycerol which is a by-product from biodiesel production is important. Generally, for every $9 \mathrm{~kg}$ of biodiesel produced, $1 \mathrm{~kg}$ of a crude glycerol by-product is produced. There is a large amount of crude glycerol on the market available for very low price due to the rapid increase of biodiesel production [1.2].

Glycerol can be used as a carbon source for the fermentative production of 1,3-PDO (1,3-propanediol) which is a monomer of PTT(Poly(trimethyleneterephthalate). PTT is a polyester with superior stretching and stretch recovery characteristics and has various usage for textile, carpets and upholstery manufacturing [3].

Before two decades, 1,3-PDO had been produced by hydration of acrolein or hydroformylation of ethylene oxide. In early 2003, DuPont developed a commercial biological process for 1,3-PDO based on the fermentation of glucose. Such a bio-based PDO made a petroleum-based PTT to be bioplastic. In spite of energy saving of glucose based fermentation by DuPont, the cost of 1,3-PDO has been still high and its price can depend on the price of glucose [4]. The low price of glycerol due to the rapid increasing of biodiesel production makes its usage of a promising alternative carbon source be possible.

A biological production of 1,3-PDO has several advantages compared with the conventional chemical production because of low cost and more eco-friendly process. 1,3-PDO had been synthesized by various microbes such as Klebsiella pneumonia, Clostridium butyricum and Citrobactor freundii [5-7]. It was reported that the final concentration of 1,3-PDO in fermentation broth was low, ranging from 30 to $130 \mathrm{~g} / \mathrm{L}$. In addition, the fermentation broth contains a mixture of 1,3-PDO, 2,3-butanediol, glycerol, lactate, acetate, succinate and other impurities which make the downstream processing of 1,3-PDO fermentation broth be difficult [8].

The downstream processes of biologically produced 1,3-PDO includes three main steps. In the first step, microbial cells are removed by using flocculation, filtration, and centrifugation. The second step contains the primary recovery processes for the removal of impurities such as acid salts and ethanol and the evaporation of water. In this step, evaporation, solvent extraction, precipitation, electrodialysis and chromatography processes can be used. In the last step, the final purification of 1,3-PDO is carried out by vacuum distillation process [9].<smiles>OCCCO</smiles>

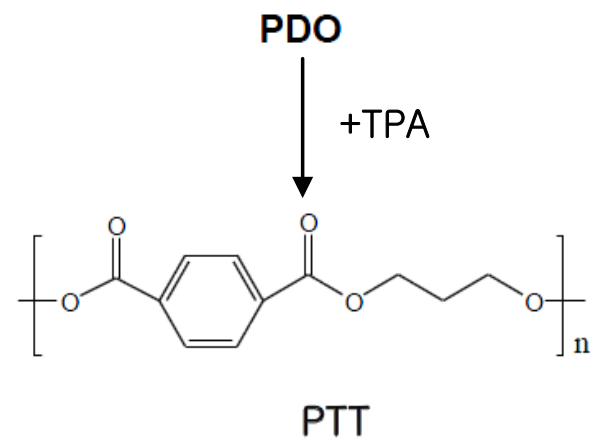

Figure 1. PTT synthesis from 1,3-PDO. 
Among these steps, the most important step is primary separation step which requires a large amount of energy for the removal of water from fermentation broth. Furthermore, the removal of acid salts from broth is not easy. For the cost effective biological production of 1,3-PDO, a low energy required separation processes with a high efficiency is essential.

For the economic removal of impurities, several processes as the primary separation step have been suggested. Broekhuis et al proposed reactive extraction by formaldehyde or acetaldehyde where 1,3-PDO was converted into a compound without hydroxyl groups and then recovered it by solvent extraction [10]. Hao et al used propionaldehyde, butylaldehyde and isobutylaldehyde as extractants for the recovery of 1,3-PDO [11]. However, the organic salts that are formed during fermentation would decline the recovery efficiency. In some cases, electrodialysis was used for the removal of organic acids salts but low product yield, membrane pollution, and high maintenance cost make this process undesirable. Recently, Hong reports that the amine-based extraction process is effective for the removal of acid salts from 1,3-PDO aqueous solutions [8].

Aqueous liquid-liquid two-phase systems are formed when two polymers or polymer/salt are dissolved together above certain concentration. The general characteristic feature of these systems is that both phases are aqueous, allowing partition of target molecules. In case of polymer/polymer or polymer/salt systems, it is difficult to use in a commercial scale due to their high cost. Therefore, for the bulk chemical such as 1,3-PDO, the cheap aqueous two-phase systems are essential.

In this study, aqueous two-phase systems composed of alcohol solvents and inorganic salts was used for the extraction of 1,3-PDO from its artificial aqueous solutions. The phase separation of two-phases containing alcohols/salt and the effect of concentration of 1,3-PDO and solvent on separation yield was investigated.

\section{Materials and Methods}

\subsection{Materials and Reagents}

1,3-PDO (Aldrich, 99.9\%) were used as received to prepare its aqueous solutions. The concentrations of 1,3-PDO ranged from $50 \mathrm{~g} / \mathrm{L}$ to $100 \mathrm{~g} / \mathrm{L}$. These concentrations were based on the concentration of practical fermentation broth produced by $K$. Pneumoniae. Hydrophilic alcohols such as methanol (Aldrich 99.9\%), ethanol (Aldrich 99.9\%) and isopropanol (Aldrich 99.9\%) were also used as received without any further purification. The salt used in this study was $\mathrm{K}_{2} \mathrm{HPO}_{4}$.

\subsection{Experimental Procedure}

For the investigation of effect of 1,3-PDO concentration, $2 \mathrm{~g}$ of $\mathrm{K}_{2} \mathrm{HPO}_{4}$ and $10 \mathrm{ml}$ of alcohol were added to the $10 \mathrm{ml}$ of 1,3-PDO aqueous solution. After stirred for $2 \mathrm{~min}$, the mixtures were held for $10 \mathrm{~h}$ at room temperature.

For the study of effect of solvent amounts, the specific concentration of 1,3-PDO aqueous solution and $2 \mathrm{~g}$ of $\mathrm{K}_{2} \mathrm{HPO}_{4}$ were added to alcohols with various volumes.

The concentration of 1,3-PDO in the top and bottom phases were measured by gas chromatography.

\section{Results and Discussion}

\subsection{Phase Diagram}

Phase diagram data are essential for the selection of optimized two-phase systems. The phase diagram was prepared by a turbidimetric titration method [12]. The concentrations of alcohols and $\mathrm{K}_{2} \mathrm{HPO}_{4}$ were calculated by the following equations.

$$
\begin{aligned}
& w_{1}=\frac{m_{1}}{m_{t}}=\frac{m_{1}}{m_{1}+m_{2}+m_{3}} \\
& w_{2}=\frac{m_{2}}{m_{t}}=\frac{m_{2}}{m_{1}+m_{2}+m_{3}}
\end{aligned}
$$

where $m_{1}, m_{2}$ and $m_{3}$ are the amount of alcohols, $\mathrm{K}_{2} \mathrm{HPO}_{4}$, and water, respectively. $w_{1}$ and $w_{2}$ are the mass fraction of alcohol and $\mathrm{K}_{2} \mathrm{HPO}_{4}$.

The bimodal curves determined at room temperature for the alcohols $/ \mathrm{K}_{2} \mathrm{HPO}_{4}$ systems were shown in Figure 2. These bimodal curves provide information about the concentrations of phase which are able to form a aqueous two-phase. Figure 2 shows that aqueous two-phase systems can be formed by adding appropriate amount of $\mathrm{K}_{2} \mathrm{HPO}_{4}$ to aqueous alcohol solutions. It is found that the ability of the alcohols for phase separation follows the order: isopropanol $>$ ethanol>methanol, which is in accordance with the order of polarity of the alcohols. From Figure 2, isopropanol has the best phase-forming abilities among hydrophilic alcohols used in this study. And Figure 2 shows that the ability of the alcohols for phase separation increased with the weight fraction of $\mathrm{K}_{2} \mathrm{HPO}_{4}$. The anion $\mathrm{HPO}_{4}{ }^{2-}$ that strongly interacts with water molecules enable to form aqueous two-phase.

In this study, partition coefficients of 1,3-PDO between two phases are as follows:

$$
K=\frac{C_{t}}{C_{b}}
$$

where $C_{t}$ and $C_{b}$ are equilibrium concentrations of 1,3-PDO in top phase and bottom phase, respectively. Extraction efficiency of 1,3-PDO can be calculated as follows:

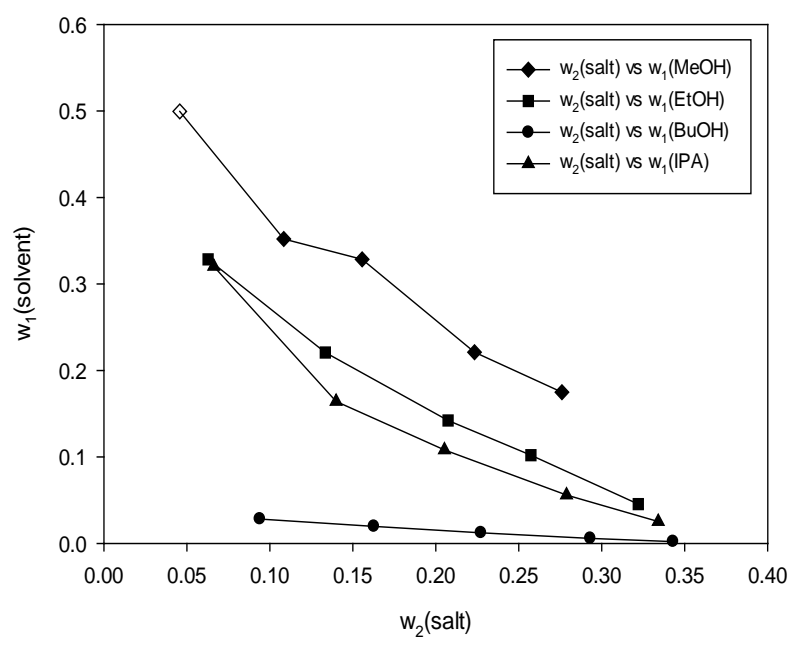

Figure 2. Phase diagram of alcohols/salt aqueous two-phase systems. 


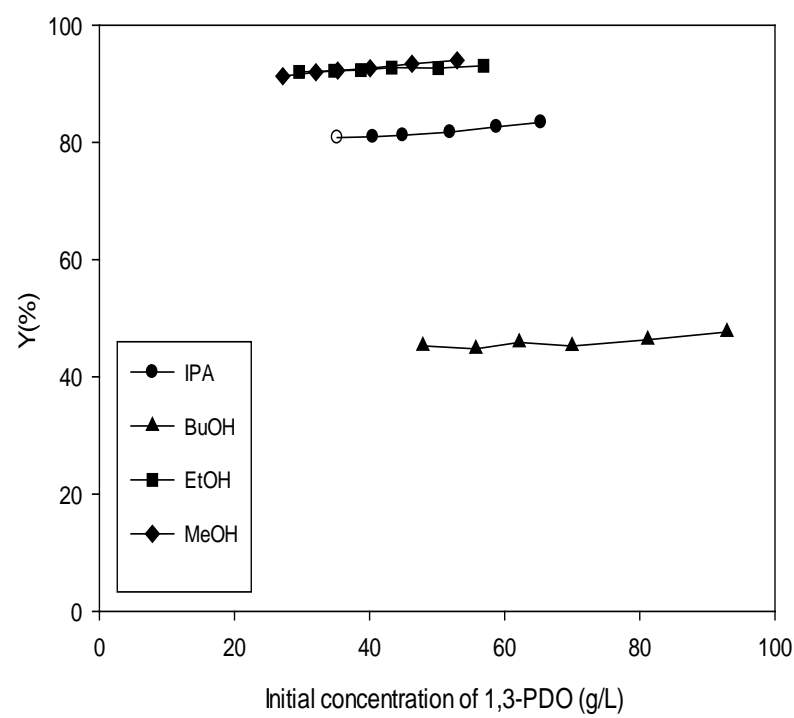

Figure 3. Effect of 1,3-PDO concentration on extraction efficiency in an aqueous two-phase systems composed of Alcohols/ $\mathrm{K}_{2} \mathrm{HPO}_{4}$.

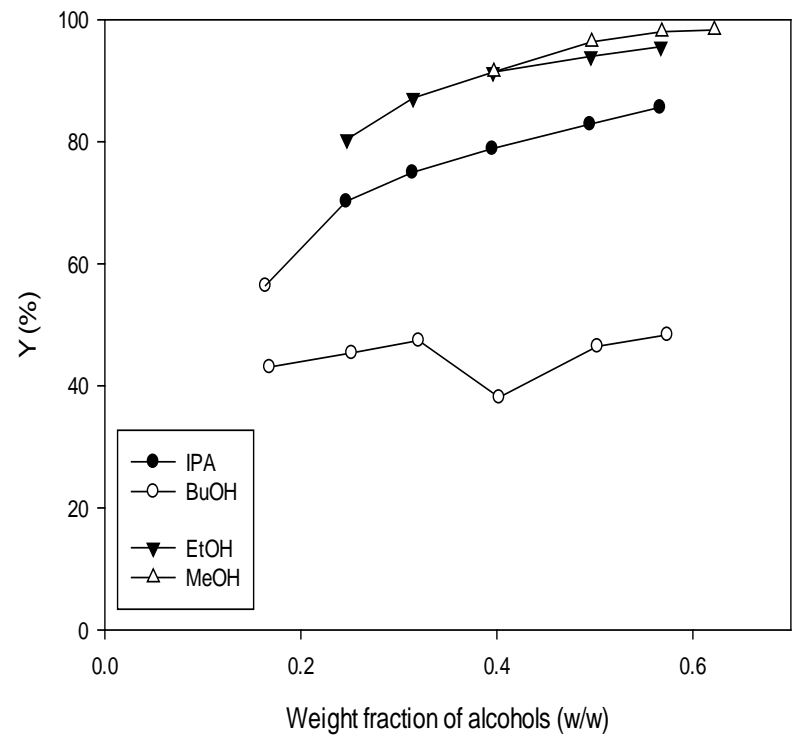

Figure 4. Effect of weight fraction of various alcohols on extraction efficiency in an aqueous two-phase systems composed alcohols $/ \mathrm{K}_{2} \mathrm{HPO}_{4}$.

$$
Y=\frac{C_{t} V_{t}}{C_{t} V_{t}+C_{b} V_{b}}
$$

where $V_{t}$ and $V_{b}$ are volume of each phase.

Figure 3 shows the effect of 1,3-PDO concentration on extraction efficiency in aqueous two-phase systems composed of various alcohols and K2HPO4. The extraction efficiency does not depend on the 1,3-PDO concentration. However, the extraction efficiency increased with the polarity of alcohols. It can be presumed that the solubility of alcohols for 1,3-PDO and the hydrogen bonding between alcohols and 1,3-PDO are proportional to their polarities. Because 1-butanol is hydrophobic, its extraction efficiency is low compared to other hydrophilic alcohols. Considering the economics of $1,3-\mathrm{PDO}$ separation processes, it is found that the only single extraction step is applicable to the primary separation of 1,3-PDO by using more than $50 \%(w / w)$ of methanol or ethanol. In case of isopropanol, multiple or continuous extraction is required.

\section{Conclusions}

In this study, the extraction of 1,3-PDO in hydrophilic alcohols/K2HPO4 was demonstrated to be effective. It is found that the aqueous two phases are easily formed with decreasing of the polarity of alcohols. However, the extraction efficiency is proportional to the polarity of alcohols. It can be concluded that methanol or ethanol is suitable for the extraction of 1,3-PDO by using aqueous two-phase. Considering the toxicity of solvents, ethanol is more suitable than methanol. Compared with other primary separation processes such as evaporation, electrodialysis and conventional solvent extraction, aqueous two-phase extraction has the advantages of low energy cost, quick phase separation and high extraction efficiency for 1,3-PDO.

\section{Acknowledgements}

This research was supported by a grant from the Academic Research Program of Korea National University of Transportation in 2012. (Hyoung Wook Lee)

\section{REFERENCES}

[1] M. A. Dasari, P. P. Kiatsimkul, W. R. Sutterlin and G. J. Suppes, "Low-pressure hydrogenolysis of glycerol to propylene glycol," Applied Catalysis A: General, vol. 281, pp. 225-231, 2005.

[2] M. Pagliaro, R. Ciriminna, H. Kimura, M. Rossi and C D. Pina, "From glycerol to value-added products," Angew. Chem. Int. Ed., vol. 46, pp. 4434-4440, 2007.

[3] R. K. Saxena, P. Anand, S. Saran and J. Isar, "Microbial production of 1,3-propanediol: Recent developments and emerging opportunities,” Biotech. Adv., vol. 27, pp. 895-913, 2009.

[4] Y. K. Hong, "Separation processes of biologically produced 1,3-propanediol,” Korean Chem. Eng. Res., in press.

[5] A. P. Zeng and H. Biebl, "Bulk chemicals from biotechnology: The case of 1,3-propanediol production and the new trends," Adv. Biochem. Eng. Biotechnol., vol. 74, 00. 239-259, 2002.

[6] C. Raynaud, P. Sarcabal, I. Meynial-Sallas and C. Croux, "Molecular characterzation of the 1,3-propanediol operon of Clostridium butyricum," Proc. Natl. Acad. Sci. USA, vol. 100, pp. 5010-5015, 2003.

[7] T. Homann, C. Tag, H. Biebl and W. Deckwer, "Fermentation of glycerol to 1,3-propanediol by Klebsiella and Citrobacter strains,” Appl. Microbiol. Biotechnol., vol. 33, pp. 121-126, 1990.

[8] Y. K. Hong, "Purification of 1,3-propanediol for production of polytrimethylene terephthalate(PTT) from biomass,” Adv. Mater. Res., vol. 320, pp. 191-195, 2011.

[9] Z.-L. Xiu and A.-P. Zeng, "Present state and perspective of downstream processing of biologically produced 1,3-propanediol and 2,3-butanediol," Appl. Microbiol. Biotechnol., vol. 78, pp. 917-926, 2008.

[10] R. R. Broekhuis, S. Lynn and C. J. King, "Recovery of propylene glycol from dilute aqueous solutions via reversible reaction with aldehydes,” Ind. Eng. Chem. Res., vol. 33, pp. 3230-3237, 1994. 
[11] J. Hao, H. J. Liu and D. H. Liu, "Novel route of reactive extraction to recover 1,3-propanediol from a dilute aqueous solution,"
Ind. Eng. Chem. Res., vol. 44, pp. 4380-4385, 2005. 